\title{
Coordinación entre el hospital y los centros de atención primaria de los pacientes en diálisis peritoneal
}

\author{
Eva Barbero Narbona, Elisabeth Tejeda Araez, Ernestina Junyent Iglesias, Nuria Pujolar Fulquet, Rosa \\ María Galí Llàcer, Lola Mendoza García de Paredes
}

\section{CAP Vila Olímpica. PAMEN, EAP Poblanou t la La Pau. ICS y Hospital del Mar. Barcelona}

\section{Introducción:}

Estudios epidemiológicos recientes han mostrado un aumento de la prevalencia de enfermedad renal crónica en España (estudio EPIRCE 9,09\% de la población general y otros estudios entre 20-35\%). La unidad de diálisis Peritoneal (UDP) atendió 44 pacientes entre 2006-12. Durante este periodo ingresaron 26 pacientes, 12 por problemas relacionados con la terapia (276 días) y 14 por otras causas triplicando el número de días ingresados (882 días). De todos nuestros pacientes el $45 \%$ eran mayores de 70 años y presentaron pluripatologias que requerían seguimiento y cuidados de enfermería. Aunque los pacientes en nuestro programa cumplieron todos los criterios de inclusión observamos que conforme pasaba el tiempo presentaban complicaciones de salud relacionadas con su patología de base o su edad que comprometían la permanencia en la técnica, así pues, nos planteamos la necesidad de coordinación con los Centros de Atención Primaria (CAP) estableciendo un protocolo de colaboración entre los profesionales de los diferentes niveles asistenciales.

\section{Objetivo:}

El objetivo del trabajo es mostrar el protocolo de coordinación con los CAP para mantener a los pacientes más tiempo en diálisis peritoneal domiciliaria (DPd) y disminuir los ingresos hospitalarios innecesarios.

\section{Metodología:}

\section{Fase 1:}

- Informar de la propuesta de colaboración con los CAP al Jefe del Servicio y a la dirección de Enfermería del Hospital.

- Conexión con la Primaria a través del Programa de Coordinación al Alta.

\section{Fase 2:}

- Reunión con los representantes de los diferentes CAP de nuestra área y formación de una comisión de trabajo.

- Formación en cuidados y signos de alarma de la DPd a los profesionales de los CAP.

- Elaboración y consenso de un protocolo común en colaboración:

- Momento de comunicación entre enfermería comunitaria/UDP.

- Perfil del paciente que necesitara intervención.

- Visitas domiciliarias a realizar por parte de enfermería comunitaria/UDP.

- Seguimiento del paciente (numero visitas hospitalarias, control telefónico).

- Momento de aviso de síntomas alarmas.

- Elaboración de una trayectoria clínica común.

\section{Fase 3:}

- Puesta en marcha de la coordinación desde enero de 2013 en los CAP y valoración a los 6 meses. 


\section{Conclusiones:}

1. La elevada prevalencia de pacientes de edad, ERC y comorbilidad, obliga a un planteamiento de coordinación entre los dispositivos de sanidad pública.

2. Esta colaboración permite el abordaje de los problemas que presentan los pacientes y su entorno (cuidador) de forma eficaz ya que se aportan conocimientos y recursos propios de ambos niveles asistenciales.

3. Alarga la adhesión a la terapia domiciliaria y mejora la calidad de vida de los pacientes.

4. Evitar la desvinculación del paciente de la asistencia primaria.

\section{Indicadores resultados}

Recoger y evaluar los resultados obtenidos en 1 año:

- Número de ingresos hospitalarios o urgencias.

- Tiempo de permanencia en la técnica excluyendo pacientes trasplantados.

- Encuesta de satisfacción, SF-12 y Zarit semestral

\section{Referencias Bibliográficas}

1. Otero A, de Francisco A, Gayoso P, García F. On behalf of the EPIRCE Study Group. Prevalence of chronic renal disease in Spain. Results of the EPIRCE study. Nefrología 2010; 30(1): 78-86.

2. Torregrosa I, Solís $M$, Pascual $B$, Ramos B, González M, Ramos C, et al. Resultados prelimina- res de la implantación de un protocolo conjunto de manejo de la enfermedad renal crónica entre atención primaria y nefrología. Nefrología 2007; 27:162-7.

3. Catalán L, Mena M, Cerdan E, Maestre S, Ascober T, Martínez I, Bueno I, Manrique J. Protocolo de actuación para el paciente con enfermedad renal crónica en atención primaria. Revista sociedad Española Enfermería Nefrológica: $n^{\circ} 4$. volumen 14.Madrid oct-dic 2011.

4. Górriz JL, Otero A. Impacto socio sanitario de la enfermedad renal crónica avanzada. Nefrología: Volumen 28. Suplemento 3. 2008: 7-15.

5. Selgas R, Aguilar J, Julián J.C, Toledo A. Realidad y futuro en el tratamiento de la enfermedad renal crónica avanzada. Nefrología: Volumen 27. Suplemento 6. 2007: 689-693.

6. Dasí N.J, Rojo E. Continuidad de los cuidados de enfermería: necesidad de coordinación entre niveles -Revista Seden 2000; n², volumen 3, II trimestre.

7. García M, Valenzuela M.P, Martínez J.C, Otero M.S. Resultados de un programa de coordinación y de información compartida entre nefrología y atención primaria. Revista oficial de nefrología 2011; 31(1): 84-90.

8. Pastor J.L, Julián J.C. Claves del proceso de información y elección de modalidad de diálisis en pacientes con insuficiencia renal crónica. Nefrología: Volumen 1.Suplemento 1. 2010; 5:15-20. 